\title{
La Notion Du Pouvoir Dans Les Institutions De Gestion De Conflits Politiques En Côte d'Ivoire: Cas De L’institution Dénommée «Le Médiateur De La République»
}

\author{
Eben-Ezer César Léonce Koffi \\ Dago Michèle-Ange \\ Amien Assémien \\ Josiane Juliette Ehui-Bla \\ Bah Mahier Jules Michel
}

Laboratoire d'Etudes et de Recherches Interdisciplinaires en Sciences

Sociales (LERISS)

Université Félix Houphouët-Boigny, Abidjan, Côte d'Ivoire

Doi: 10.19044/esj.2018.v14n8p199 URL:http://dx.doi.org/10.19044/esj.2018.v14n8p199

\begin{abstract}
The study of conflict management, especially that relating to mediation, occupies an important place in the advent of conflicts in traditional African environment. In the 1990s, it was introduced gradually into the Ivorian institutions to answer socio-political excesses. However, despite its existence, this institution has faced several oppositions in regards to the aim of the study. This paper focuses on identifying the sociological contours that emerge around the dynamics of mediation in relation to power in the recent conciliatory context. On the basis of direct observations of semi-directive interviews, this research examined the report of the executive on the institution in the conciliatory process. The paper analyzes that the dependence of this institution on the executive power leads to dysfunction of the institution as a result of the reconciliation process.
\end{abstract}

Keywords: Conflict, Mediation, Power, Politics System

\section{Résumé}

L'étude de gestion des conflits, notamment celle relative à la médiation occupe une place prépondérante dans l'avènement des conflits en milieu traditionnel africain. Si bien que celle-ci a été introduite progressivement, dans les années 1990 dans les institutions ivoiriennes, pour répondreaux débordements socio-politiques. Cependant, malgré son existence, cette 
institutionsembleêtre en opposition à sa visée depuis peu. L'objectif de cet article est de cerner les contours sociologiques qui se dégagentautour de la dynamique de médiation en rapport avec le pouvoir dans le contexte conciliatoire qui prévaut depuis peu. Sur la base d'observations directes, d'entretien semi directif, ces recherches ont examiné le rapport qu'à l'exécutif sur l'institution dans le processus conciliatoire. Il en découle des analyses que la dépendance de cette institution au pouvoir exécutif entraine un dysfonctionnement de l'institution dans le processus de réconciliation.

Mots-clés: Conflits, Médiation, Pouvoir, Système Politique

\section{Introduction}

La récurrence des conflits politiques violents en Afrique représente l'un des intérêts majeurs des Etats. De sorte que leur gestion est devenue le point focal de règlement consacré par la Charte des Nations Unies. En effet, cette disposition qui érige en règle juridique l'obligation des Etats à négocier pour trouver une solution à leur problème, se base sur le fait que les parties en conflits,dont le désaccord est susceptible de menacer la paix et la sécurité internationale, doivent en rechercher la solution, avant tout, par voie de négociation, d'enquête, de médiation, de conciliation, d'arbitrage, de règlement judiciaire, de recours aux organismes ou accords régionaux, ou par d'autres moyens pacifiques de leur choix (Déclaration de Manille, volume 28, 1982, p. 618). Au regard du milieu africain qui referme un caractère conflictuel (putsch, violence, instabilité politique, opposition latente ou manifeste) bien des mécanismes ont été élaborés en vue de résoudre les conflits sociaux. C'est dans cette perspective que l'Union Africaine s'est engagée à œuvrer pour la promotion de la paix, de la sécuritéet de la stabilité dans le continent, conformément auxprincipes de la Charte des Nations Unies (article 4 (e) et 4(f) de son acte constitutif). La Côte d'Ivoire, pays membre de l'UA (Union Africaine) s'est inscrite dans cette dynamique en se dotant d'un organe de conciliation dénommé «Le Médiateur de la République ».

Créée le 29 septembre 1995, dans l'optique de résoudre le problème de la succession du Président Félix Houphouët-Boigny, la Médiation ivoirienne a été érigée par décret en opposition à la constitution ivoirienne du 03 Novembre 1960. Le décret de création met en place non pas un Médiateur mais plutôt un Président, d'où l'organe présidentiel de la médiation, (OPREM) qualificatif, marquant la dépendance de l'exécutif. Sa mission est de régler les différends, les divergents de l'administration, mais aussi de contenir les débordements politiques, et de réparer en équité l'appareil gouvernemental et les torts socio-politiques. Cependant, depuis sa création, ses différentes actions relatives à la réconciliation nationale ont été toujours orientées par le pouvoir politique ou ont été fait par l'entremise du Président de la République. Alors 
que l'institution se caractérise comme étant un organe apolitique. Garant de la cohésion sociale, de par ses attributs, la Médiation ivoirienne a contribuée à la recherche des solutions relatives aux différents conflits qui se sont succédé au fil du temps dans l'histoire politique du pays. Ainsi partant du forum de réconciliation Nationale avec le comité de Médiation pour la réconciliation nationale (CMRN) en 2000 jusqu'à la gestion du referendum en 2002 par la Commission Consultative Constitutionnelle et Electorale (CCCE), il en ressort que malgré les actions de l'exécutif sur l'institution de nombreuses crises continue d'émailler la vie socio-politique du pays et plonge le pays dans une impasse, marqué, çà et là par la crispation communautaire, l'insécurité, la peur d'un nouveau conflit etc...

Suite à ces différents constats cités plus hauts, il incombe de savoir qu'est ce qui explique les pratiques du pouvoir exécutif dans le processus de réconciliation ? L'action du pouvoir exécutif dans le champ de Médiation estelle légitimée? A quelle fin répond l'instrumentalisation de la Médiature Ivoirienne dans le système politique ?

L'objectif de cette étude est de comprendre les logiques sociopolitiques qui entravent le fonctionnement de l'institution dénommée «Le Médiateur de la République » dans le processus conciliatoire qui prévaut en Côte d'Ivoire. L'hypothèse que l'on propose est que la dépendance de la Médiation au pouvoir exécutif entraine un dysfonctionnement de l'institution dans le processus de réconciliation.

La démarche s'articule autour de trois (3) axes. A savoir, l'action du pouvoir exécutif dans le champ de Médiation (i), l'appui technique des autorités nationales et internationales en charge dans le processus de réconciliation (ii), et l'instrumentalisation de l'organe de Médiation (iii).

\section{Méthodologie}

La méthodologie utilisée dans cette recherche nous a permis de conduire notre enquête de terrain dans le District d'Abidjan.Le choix de ce site se justifie par le fait que le siège principal de la Médiature y est et aussi par le fait que toutes les institutions de la République y sont concentrées. La concentration de ces différentes institutions politiques et administratives a été pour nous un atout favorable, car cela nous a permis de faire une triangulation avec les autres entités qui se déploient dans le même champ d'action de notre étude. Les entretiens ont été menés avec le personnel de la Médiation ivoirienne, mais aussi avec les membres de la société civile qui travaillent de concert avec la Médiation, puis les agents de la CDVR (Commission, Dialogue, Vérité et Réconciliation), devenu CONARIV (Commission National pour la Réconciliation et de l'Indemnisation et des Victimes) au fil du temps, des agents du PNCSpuis les membresde certainspartis politiques. Ces acteurs ont été choisis sur la base de leur position hiérarchique et de leur 
niveau d'implication dans le processus de réconciliation. Ils sont entre autres, chef de cabinet, conseiller du Médiateur, chargé de communication à la Médiation, Chefs de villages solliciter par l'institution pour leur expertise coutumière, responsable du service de gestion des crises, personnel d'ONG (Organisation Non Gouvernemental) qui travaille en synergie avec l'institution, et membres de partis politiques. Au total trente-quatre (34) individus ont été interviewés.

Ainsi pour mieux appréhender la étude, il nous a semblé probant d'utiliser l'analyse de contenu et l'analyse systémique. L'analyse de contenu textuel et thématique pour décrypter les discours des enquêtés et en dégager un corpus de mots, tout en les codant selon les catégories qui se sont construit et améliorés tout au long de l'étude. Et ce, dans l'optique de comprendre les sens et les perceptions que chaque acteur donne à son action. Puis l'analyse systémique, qui a consisté à nous appuyer sur les rapports et les liens d'interdépendance qui existent entre les différentes entités. Ainsi la perspective d'entreprendre une étude scientifiqueportant sur un tel sujet, somme toute,sur les conflits et la manière de les gérer nous a amené à mobiliser la théorie de domination de Bourdieu (2004), et la théorie de l'instrumentalisation d'Yveline Dévérin (2006). Partant de ces postulats, l'utilité de la théorie Bourdieusienne, pour notre travail se situe à deux niveaux. Dans la mesure où elle nous permet, tout d'abord, d'établir un lien entre les différents champs dans lesquels se dépoilent les acteurs, puis d'en déceler les différentes positions occupées par les acteurs politiques (Président de la République, Président de l'Assemblée National, Médiateurs, CSCI, PNCS, Agents de la CONARIV ex CDVR, institutions annexes, membres de partis politiques). De plus, elle nous permet de comprendre l'impact généré par le positionnement des acteurs détenant le pouvoir politique en tant qu'acteur dominant sur les agents de la Médiation Ivoirienne en particulier. Et ce, de par les différentes ressources (capitaux économiques, social et politiques) qu'ils mobilisent pour légitimer leurs actions. Quant à la théorie d'Yveline Dévérin, elle nous a permis d'enrichir encore plus la théorie Bourdieusienne des capitaux puis d'expliquer comment s'effectue l'instrumentalisation de l'institution par le pouvoir politique.

\section{Résultats}

Cette partie est consacrée aux logiques socio-politiques qui entravent le fonctionnement de l'institution Ivoirienne dans le processus conciliatoire qui prévaut depuis peu dans le pays. Pour ce faire, elle va prendre en compte d'une part l'action du pouvoir exécutif dans le champ de Médiation etd'autre part l'appui technique des autorités nationales en charge dans le processus de réconciliation, sans toutefois omettre l'instrumentalisation du pouvoir exécutif dont fait l'objet cette institution. 


\section{Contexte de Création et Fonctionnement de la Médiation}

L'idée d'une Médiation à émerger en Côte d'Ivoire entre 1993 et 1995, ou diverses tensions ont émaillé la vie socio-politique de ce pays. Ainsi transposé dans un cadre étatique, il faut dire que la Médiation est une notion en vogue et apparait comme un M.A.R.C, c'est-à-dire un Moyen Alternatif de Résolution des Conflits, qui est moins onéreux.

Vu l'importance d'une telle instance dans le système de résolution des conflits, la proximité entre les différents acteurs et les coûts qui sont moindre, la Médiation devient une option palliative qui est au cœur des mécanismes et modes de gestions de conflits. C'est à juste titre qu'un enquêté affirme que :

«La notion de Médiation est à la mode ces derniers temps, elle existe maintenant dans la plus part des pays Africains et est un moyens efficace de résolution des conflits qui nécessite pas assez de moyens et de financements comme on le voit pour d'autres institutions » (K.F. Agent à la Médiature).

Vu sous cet angle, il faut dire que la résolution de conflit entraine dans bien des cas des dépenses excessives lorsque le conflit est effectif ou se déclenche. Ainsi pour éviter cela, bons nombres d'acteurs préconisent le mode préventif de la Médiation. Cela a été lors de la crise concernant la succession de Président Houphouët-Boigny en 1993. Le témoignage suivant met en évidence cela :

"Aussi, il faut dire qu'en Côte d'Ivoire, la Médiation a été un moyen préventif que curatif qui dans bien de cas, a permis de circonscrire les tensions socio-politiques du moment. Surtout quand le Président Houphouët est mort, on a frôlé le pire hein. Grâce à la constitution ». (H.G. Conseiller du Médiateur)

A ce niveau, il faut dire que la Médiation utilise les textes juridiques pour effectuer ses différentes actions.

\section{L'action du Pouvoir Exécutif dans le Champ de Médiation}

Concilier, réconcilier, arbitrer, juger, régler, trouver un compromis, autant de mot qui désigne le règlement à l'amiable d'un conflit, d'un litige entre les parties. Ainsi, légitimée par les lois et règles établies par la constitution ivoirienne, la Médiation a pour rôle classique de résoudre les problèmes de dysfonctionnement de l'administration, c'est-à-dire les problèmes entre les administrés et l'administration. Mais aussi les problèmes relatifs à la cohésion sociale. En effet, plusieurs actions ont été menées dans l'optique de raffermir la cohésion sociale, mais cela a été possible que par l'entremise du Président de l'époque, alors que cette institution de par ces textes portant sa création stipule qu'elle est une instanceindépendante. Et cela se perçoit en ces termes: 
«La Médiature est une institution apolitique, maisquand le Président de la République a créé la structure qui s'appelle le CMRN, il lui a confié la présidence à la Médiature. De même que le forum de réconciliation nationale. Vous savezle Président de l'époque, nous a confié le suivi de tout le processus. Mais à vrai ce n'est pas notre rôle. Bon, comme c'était une branche de la Présidence en son temps c'est pourquoi le Médiateur a fait ce que le Président lui demandait. Sinon les textes disent que la Médiature est indépendant et que la Médiature peut s'autosaisir » (I. K. agent à la Médiature).

Ces propos laissent entrevoir l'action du pouvoir exécutif dans les opérations que la Médiation. Cela, se perçoit tout de même à travers ce discours :

«La Présidence, nous a confié la commission consultative constitutionnelle et électorale (CCCE), afin que nous nous penchions sur le problème de légitimité des candidats politiques à la course au pouvoir, et aussi de tous ceux qui dorénavant aller se présenter comme candidat. Cela, lui tenait à cour puisque cela était un challenge pour lui » (H.G. Conseiller du Médiateur).

Alors les textes stipulent que la Médiature est en mesure de s'autosaisir dans le cas où la cohésion sociale est mise en mal. En effet, les enquêtes montrent qu'avant la loi organique $\mathrm{N}^{\circ} 2007-540 \mathrm{du} 1^{\mathrm{er}}$ Août 2007, l'instance dénommée le Médiateur de la République n'avait pas pleine autorité, plein exercice de ses fonctions. C'est dire que depuis sa création jusqu'à la mise en application de la loi organique du $1^{\text {er }}$ Août 2007, la Médiation ivoirienne n'avait ni règles, ni lois figées qui devait servir de balise, de boussole dans la gestion de conflit. Alors que le renouvellement de cette instance différait déjà de 1'OPREM.Les différents rapports-bilans 2003-2007 de la Médiature, stipule que :

«C'est à l'Assemblée générale des ombudsmans Médiateursque le Médiateur Mr. M.E s'est rend compte qu'il n'avait pas une pleine qualification pour exercer en tant que Médiateur proprement dit. p.57

En effet, il en découle des lectures que c'est après cette assemblée que l'institution a été dotée d'une loi organique fixant les modalités et le fonctionnement de l'institution. Le problème de légitiméainsi résolu, le constat est que les actions de la Médiation Ivoirienne se sont toujours fait par l'entremise du Président du moment. Nous pouvons citer pêle-mêle l'intervention de la Médiature à Duékoué, l'intervention de la Médiature à Bondoukou pour le cas de succession de Chef, etc.

C'est dire que le pouvoir exécutif est au cœur de cette institution. De même, le constat est que la Médiature Ivoirienne ne peut aussi exercer librement sans que le pouvoir exécutif ne soit au cœur de ces actions. Puisque, chaque année Le Médiateur de la République présente les rapports d'activités 
de son Institution au Président de la République. D’où une prédominance de l'exécutif sur l'institution.

\section{L’appui Technique des Autorités Nationales et Internationales en Charge dans le Processus de Réconciliation}

Dans le souci de trouver des solutions immédiates aux problèmes de disfonctionnement du corps social, plusieurs alternatif ont été posé. D'où la création d'une commission et d'autres organes rompus à cette tâche. A ce sujet les enquêtes révèlent que la création de nouveaux organes dans le champ Médiatique relève d'une volonté politique. Et cela on peut le percevoir en ces termes:

"L'idée d'une commission de réconciliation et de programme nationale de Cohésion sociale résulte d'une volonté politique et est entériné par les pouvoirs publics et les décideurs internationaux ». (H.G. Conseiller du Médiateur)

C'est dire que le pouvoir en place n'est pas le seul arbitre qui prend les décisions, mais se réfère ou se soumet dans bien des cas à un Etat supranational puisque un Etat ne peut vivre en autarcie.

En nous basant sur les recherches de Vicent Briant et Yves Palau (2005) qui relève de la théorie constructiviste et organiciste nous avons tenté d'expliquer le phénomène de la Médiation en la modélisant de la façon suivante:

Figure 1. système de relation de la Médiation

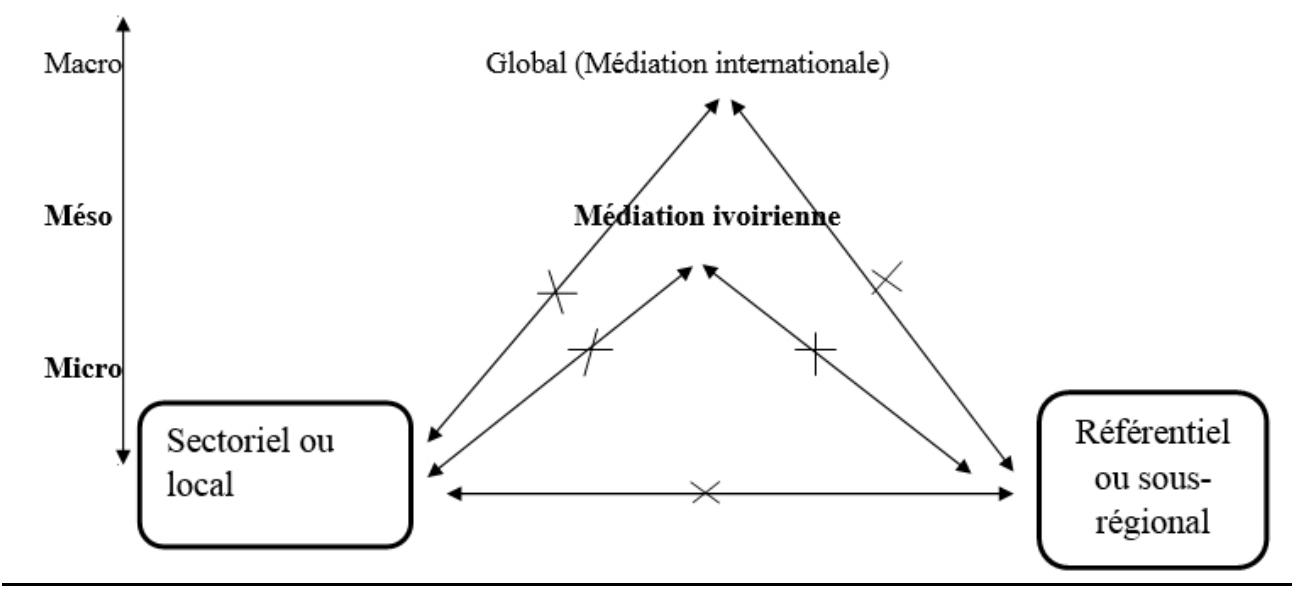

\section{Interprétation}

- Le local et le sous régional sont les milieux oùla Médiation s’effectue, et représentent le niveau micro du système. Ici cette Médiation est purement traditionnelle.

- La Médiation ivoirienne, quant à elle, représente le niveau méso. Elle est hybride, du fait qu'elle utilise les procédés découlant des mécanismes de 
résolutions traditionnelles ivoiriens, mais aussi des mécanismes de l'ombudsman provenant de l'extérieur.

- Le global ici, renvoie à la Médiation internationale, c'est le niveau macro de la chaine. Tous ces différents niveaux sont imbriqués, de sorte que cela forme un tout.

- Les flèches à double sens signifient que la mise en relation est bilatérale.

- La croix (X) désigne, le fait que la Médiation ne peut être possible directement et montre aussi qu'elle peut être rompue si toutefois les parties n'ont pas trouvé de consensus.

Somme toute, nous pouvons noter que les Médiations ne fonctionnent pas de manière individuelle. Elles sont complémentaires les unes des autres, et montrent comment la réalité sociale est un tout qui s'enchevêtre les uns dans les autres. $\mathrm{Vu}$ sous cet angle, la Médiation s'avère être un ensemble d'instances politiques et institutionnelles dont la décentralisation est un principe subsidiaire. D'où une stratification de la Médiation au niveau micro, méso et macro du système social. C'est dire que la Médiation de par ses attributs entretient des rapports de collaboration avec les structures locales et internationales. De même qu'au sein des différents niveaux. Pour ce qui concerne, la Côte d'Ivoire, il faut dire quela Médiation entretient des rapports avec d'autres institutions, telles que l'Assemblée Nationale, la Présidence de la République. C'est ce que nous avons tenté d'expliquer dans la Figure suivante.

Figure 2. Système de la Médiation au pouvoir exécutif en Côte d'Ivoire Pouvoir exécutif

Assemblée Nationale

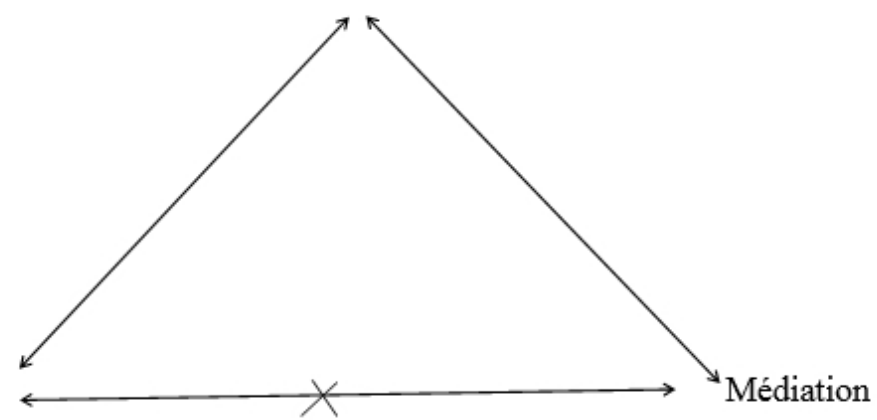

\section{Interprétation} bilatérale.

Les flèches sont à double sens pour signifier que la mise en relation est

La croix X désigne le fait que l'Assemblée Nationale peut octroyer ou pas le pouvoir d'injonction à la Médiation Ivoirienne.

Ici, la Médiation s'apparente à une structure gouvernementale à qui l'exécutif délègue son pouvoir à un moment donné. Mais aussi, comme une 
entité qui constitue un véritable enjeu pour l'exécutif. De sorte, la Médiation ivoirienne ne peut agir seul.

C'est en se basant sur ses différents postulat qui régit le mode de Médiation que se fonde les interventions supranationales. C'est dans cette optique qu'à la suitedu ballet de Médiateurs conjoint de la CEDEAO (Communauté économique des Etats d'Afrique de l'Ouest) et de l'UA (Union Africaine) que va être crééla commission, dénommé CDVR (commission dialogue vérité et réconciliation et par la suite le PNCS (Programme National de Cohésion Sociale).

\section{L'instrumentalisation de L'organe de Médiation Par le Pouvoir Politique}

La contribution particulière à mobiliser l'instance de la Médiation au plan politique consiste à dissimuler les intérêts spécifiques du pouvoir. Cette perspective est liée à une vision contemporanéiste d'être encore dans l'arène politique, sans toutefois posséder la majorité du pouvoir. Ainsi, la Médiation devient une institution annexe, permettant de construire le pouvoir. Les propos suivant illustre cette logique:

« Le Médiateur est nommé pour six (6) ans alors que le Président pour cinq (5) ans, cela est un avantage pour nous, car nous pouvons finaliser notre travail et faire pression si nécessaire lorsque les choses ne tournent pas comme, nous le voulons, c'est-à-dire si le pouvoir change » (Mme A, chef de cabinet du Médiateur).

Ce positionnement stratégique permet au Médiateur d'influencer le pouvoir en place si toutefois le Président de la République n'est pas du même bord que ce dernier. Ou, comme nous l'avons vu plus haut, facilite l'exécution des décisions venant du pouvoir exécutif.

Ainsi en s'appuyant sur le positionnement stratégique des Médiateurs dans la sphère politique, on peut ainsi dire que la Médiation est l'expression de configuration des rapports entre les individus, entre les groupes de personnes à un moment donné. Elle est un système de pouvoir administratif et politique qui dans bien des cas est légitimé, et permet d'arriver à des fins politiques. De ce faite, on constate que le phénomène en lui-même est une construction sociale qui permet aux différents acteurs dese maintenir au pouvoir ou d'accéder au pouvoir. C'est qui s'illustre à travers ces propos:

«En confiant la CCCE (Commission, Constitutionnelle, Consultatif et Electoral à la Médiature, le Président de ce moment a voulu que la conjonction « et » l'emporte sur la conjonction « ou » pour faciliter les choses, de sorte qu'il n'y pas crise ».

Dans un tel contexte social, la Médiature se départi de ses enjeux et ses intérêts politiques pour se pencher sur l'intérêt commun et rappelle la communauté à la cohésion sociale. Cependant, en dépit de ces faits certains 
acteurs politiques affirment qu'ils n’ont pas confiance au Médiateur. Cela se perçoit à travers ses différents propos qui suivent:

"Le Médiateur a été nommé par mon adversaire politique, je ne sais pas pourquoi je vais l'écouter, il ne dit que ce qui l'arrange lui, mais encore plus ce qui arrange son parti politique ». (M. K.G. militant d'un parti politique).

Cette affirmation dénote de la non légitimité de la Médiation par les acteurs politiques. En particulier des membres des partis politiques. De plus cette assertion de $\mathrm{Mr}$. D, chargé de communication à la Médiature, montre que l'institution ne revêt pas toutes les attributions pour exercer librement, ni de pouvoir de coercition. Il affirme:

«Celui qui t'a nommé il faut qu'il t'habille d'un pouvoir réel pour que tu puisses exercer pleinement ta fonction. Or il y'a des problèmes auxquels la Médiature ne peut pas faire face à cause de la loi organique. En plus on constate que dans toutes nos institutions, le politique a pris le pas sur des missions, ce qui fait que nous avons du mal à être reconnu par les différents acteurs politiques ».

Cela traduit la perception idéologique que lespartis politiques ont avec la Médiature. Ce qui dénote une crise de reconnaissance des partis adverses. De même, il en ressort que le pouvoir en place donne des attributs à une institution selon les enjeux du moment. Cette façon de s'approprier l'institution pour des enjeux d'ordre politique, social et économique, fait ressortir un problème crucial dans la compétition politique des différents partis.

\section{Discussion}

Dans cette discussion vise à faire un dépassement de tous les aspects marquants que nous avons pu relever tout au long de cette recherche. Ainsi, afin de rendre plus fluide cette analyse, nous nous appuierons essentiellement sur les résultats les plus significatifs qui ont une connotation sociologique. Ainsi, Il va s'agir, d'analyser le système dans lequel la Médiation ivoirienne évolue, puis de nous atteler sur la dépendance de la Médiation ivoirienne au pouvoir exécutif. Sans toutefois omettre la manifestation du politique dans les institutions.

\section{L'action du Pouvoir dans le Champ de la Médiation}

La société est une sorte d'immense corps dont les sous éléments sont les organes qui prennent sens, que par rapport à l'ensemble du corps. Et comme un Etat ne peut vivre en autarcie, elle se doit d'intégrer cet ensemble du corps, d'où le système de Médiation internationale auquel la Médiation Ivoirienne appartient. Cette imbrication des différents Etats dans la gestion des conflits relève des relations internationales qui permettent de structurer les 
rapports supranationaux. Selon la théorie collectiviste de Vicent de Briant et Yves Palau (2005), le corps social dans lequel la Médiation baigne devrait être le contre-pouvoir de l'Etat et permettre de réguler le dysfonctionnement de celle-ci. Cependant, l'on constate qu'en Afrique et par ricochet en Côte d'Ivoire, la Médiation est plutôt un prolongement voir la continuité du pouvoir et par surcroit un prolongement du parti politique exerçant son pouvoir sur tout le corps social. En plus de cela, il faut ajouter que la Médiation Ivoirienne est fondée sur le principe de maintien d'unité sociale, plutôt que sur une volonté purement stratégique. Cette assertion n'est pas du tout fondée pour Fathi Ben Mirad(2002) puisqu'il perçoit la Médiation autrement. Pour lui, la Médiation n'est fondée que sur des conceptions qui traduisent une vision commune, puisqu'elle est le produit de positionnement et d'opinions multiples et variées, traversée par les enjeux de reconnaissance symbolique et professionnelle. C'est dans cette optique que, la Médiation Ivoiriennea été créée. C'est-à-dire dans un esprit de consolidation del'unité nationale, tout en l'utilisant comme moyen pour accéder à des fins politiques. Ainsi, la reconnaissance politique au sein du système de Médiation lui confère sa légitimité, puisque c'est sur cette légitimité qu'elle s'appuie pour mener ces actions. Il faut ajouter tout de même que la plupart des Médiations sont des circuits économiques, comme le relève Yveline Dévérin (2006) dans sa critique du bon usage de la Médiation. Si donc l'économie se mène à la politique, il faut s'en soit dire que cette sphère renferme, autant d'enjeux qui font que les médiations, et par ricochet la Médiation Ivoirienne est imbriquée dans un système où la domination est de mise. A ce niveau, la Médiation se trouve réduite à une instance dépendante parmi d'autres, de sorte que tout le système qui se développe autour en lui-même, forme une nouvelle architecture institutionnelle basée sur le principe organicisme.

Au regard de nos différentes observations, il apparait que la Médiation est enserrée dans un ordre étatique et son autonomie dépend purement de sa capacité d'être financé par les pouvoirs en place, mais aussi par les pouvoirs " occidentaux » d'où l'action du pouvoir politique dans le champ de la Médiation. De même que l'action des différents organismes supranationaux dans les prises de décisions de la Médiation Ivoirienne.

\section{Les Différentes Relations du Système}

Dans l'optique de maintenir l'ordre établi par le système organisationnel dans lequel elle baigne, la Médiation ivoirienne est dépourvue de pouvoir de coercition. Cela est marqué par le fait que la Médiation n'arrive pas à faire appliquer les différentes décisions sur le corps social. De même, il en ressort que dans le traitement des problèmes administratifs ne répondent pas aux invitations du Médiateur. Selon Abel Richard (2014), cela s'explique par le fait que la Médiation Ivoirienne est un mode de justice informelle qui 
repose sur des leurres dans la mesure où il est dépourvu de coercition, puisque celle-ci utilise des moyens souples de domination pour résoudre des problèmes qui sont d'une importance capitale. Pour Denis- Constant Martin (2010), le fait que, la Médiation s'apparente à un phénomène qui émerge de la société civile, c'est dire qui surgit du bas vers le haut, cela devrait lui permettre d'acquérir le pouvoir nécessaire pour mener à bien ses actions. Cependant dans notre cas de figure, la Médiation ivoirienne n'est pas le produit d'une volonté collective. Mais plutôt le fruit d'une minorité d'individus susceptible de résoudre le problème de dysfonctionnement politique, découlant de la succession du Président de la République. Cette initiative devient avec le temps, un cadre institutionnel de gestion des conflits rattaché à la présidence de la République, d'où son manque de pouvoir de coercition. Ceci s'explique par le fait qu'elle est liée au pouvoir exécutif, qui a, la capacité de lui conférer plein pouvoir, selon le contexte politique du moment. Sous cet angle, on peut donc en déduire que la Médiation Ivoirienne n'est pas indépendante comme le stipule son article $23 \mathrm{du}$ titre VI relatif à son fonctionnement de la loi organique de 2007. Ce qui explique que les actions qu'elle a eu à mener de 1999 à 2002 ont été faites par l'entremise du chef de L'Etat. Cette idéologie dénote d'une volonté politique qui permet au pouvoir d'utiliser la Médiation Ivoirienne selon ses intérêts et les enjeux du moment.

\section{Le Politique dans les Institutions}

Selon, Delphine DULONG (2012) l'institution politique des régimes démocratiques (parlement, assemblée national, présidence...) ont un pouvoir normatif à nul autre pareil qui leur permet de classer, d'organiser, et même d'officialiser l'existence des groupes sociaux et leurs différents activités. De même que les institutions annexes leur permettant de construire leur autorité. C'est donc ce postulat qui guide la plupart des institutions démocratiques. Et donc qui légitime leur action dans la plupart des institutions annexes telles que la Médiation, la société civile... Ainsi au lieu d'aider à mieux comprendre le fonctionnement des institutions et d'établir une coopération stable et pacifique en leur donnant plein pouvoir, l'on constate une mobilisation de ses institutions dans le fonctionnement du jeu politique. Ce positionnement peut paraitre incongru pour certains partisans dans la mesure où les textes qui régissent ces institutions parlent d'indépendance. Mais, de quelle indépendance parle-t-on lorsque, il y'a une instrumentalisation de ses différentes institutions?

Dans un système ou le politique a pris le dessus dans les instances, l'indépendance vis-à-vis du pouvoir exécutif devient parfois une utopie. Car, comme le constate, la Médiation existe et la politique des autres instances qui se déploient dans le même champ d'action que la Médiation la défavorise dans certaines actions. Si l'on veut s'en tenir au fait que la Médiation est un terme 
plus fort que la réconciliation, la négociation et même la cohésion sociale, il n'en est pas moins raisonnable de l'impliquer dans la gestion des conflits, puisque c'est l'un de ses fonctions.

De façon générale, une première constatation montre que le pouvoir exécutif domine la sphère de gestion des conflits. La seconde constatation est que la Médiation Ivoirienne est dépourvu de pouvoir de coercition et la troisième est que le politique dans bien de cas domine les rapports dans l'arène institutionnelle et est perçue comme un facteur majeur qui entrave le fonctionnement de l'institution dans le processus de réconciliation en Côte d'Ivoire.

\section{Conclusion}

Concilier, réconcilier, arbitrer, juger, régler, trouver un compromis, mettre d'accord, autant de mots qui désigne le règlement amiable d'un conflit. Ainsi, il a été question pour nous de montrer à travers cette étude que la Médiation Ivoirienne est imbriquée dans une sphère politique qui dans bien de cas, étouffe son pouvoir. Ainsi, les résultats et les analyses qui précédent ne prétendent pas combler un quelconque vide, mais tente de relever l'intérêt particulier du contexte, du caractère spécifique et sociologique des modes de gestions des conflits en Côte d'Ivoire. En effet, il a été pour nous de montrer les représentations et la structuration des rapports de la Médiation de la République. Tout en montrant la dépendance de la Médiation au pouvoir exécutif, en relevant les facteurs qui sous-tendent l'implication du politique dans l'arène de Médiation, et par ricochet dans les institutions Ivoiriennes.

Somme toute, ce procédé semble participer à la préservation des liens sociaux, mais renferme des écarts que le politique se doit de répondre. Cela dit, nous pouvons nous interroger sur la nécessité d'implanter plusieurs instances de Médiations dans les régions de la Côte d'Ivoire, de sorte à rapprocher cette instance de la population et de créer des structures de veille, en vue de freiner les conflits socio-politiques.

\section{References:}

1. Abel Richard (2014). The politics of informal justice, volume (n 2) : comparative studi

2. Ben Fathi (2002). Sociologie des pratiques de Médiation, collection l'Harmattan.

3. Denis-Constant Martin (2010). L'identité en jeux politique, identification, mobilisation Karthala, coll. «Recherches internationales »

4. Delphine Dulong (2012). Sociologie des institutions politiques, collection repères : Sciences politiques, droit. 
5. Guillaume d'Alancon (2006). Surmonter le conflit: les racines philosophiques de la médiation. librairie téqui.

6. Léopold Senghor, S. (1957). Discours prononcé lors de la conférence africaine de l'indépendance.

7. ONU (Organisation des Nations unies). «Origine des gestions de conflits»,http//www.un.org, consulté le 29/07/2014.

8. Pierre Bourdieu (2004). Esquisse pour une auto-analyse, Paris, Raisons d'Agir, collection Cours et travaux.

9. Six Jean-François \& Mussaud Véronique (2006). La Médiation, seuil.

10. Thuderoz, G. (2008). «Sociologie du conflit et de la négociation sociale », http//www.wikipédia.com, consulté le 04/08/2014

11. Vicent Briant \& Yves Palau (2005). La Médiation : définition, pratiques et perspectives, collection Armand Colin.

12. Yvéline Dévérin (2006). «crise ivoirienne: du bon usage de la Médiation », www.pambazuka.org/fr, consulté le 29/09/2014 